\title{
Expression of vimentin filaments in canine malignant mammary gland tumors: A simulation of clinicopathological features of human breast cancer
}

\author{
SANAZ RISMANCHI $^{1}$, ORLY YADEGAR ${ }^{2}$, SAMAD MUHAMMADNEJAD $^{3}$, \\ SAEID AMANPOUR ${ }^{1}$, MASOUD TAGHIZADEH-JAHED ${ }^{4}$ and AHAD MUHAMMADNEJAD ${ }^{5}$
}

\footnotetext{
${ }^{1}$ Cancer Models Research Center, Cancer Institute of Iran, Tehran University of Medical Science, Tehran 1419733141;

${ }^{2}$ Faculty of Specialized Veterinary Sciences, Science and Research Branch, Islamic Azad University, Tehran 14515/775;

${ }^{3}$ Research Center for Molecular and Cellular Imaging, Tehran University of Medical Sciences, Tehran 1419733141;

${ }^{4}$ Reproductive Biotechnology Research Center, Avicenna Research Institute, ACECR, Tehran 1177-19615;

${ }^{5}$ Cancer Research Center, Cancer Institute of Iran, Tehran University of Medical Science, Tehran 1419733141, Iran
}

Received May 16, 2014; Accepted June 13, 2014

DOI: $10.3892 / b r .2014 .312$

\begin{abstract}
Canine malignant mammary gland tumors (CMMGTs) are the most common malignancies observed in females. Several biological similarities have been reported between CMMGTs and human breast cancer (HBC). The present study aimed to assess the correlation of vimentin filaments overexpression, as part of the process of epithelial-mesenchymal transition (EMT) and the clinicopathological characteristics in CMMGTs. The clinicopathological characteristics of 42 CMMGTs were collected. Paraffin-embedded blocks underwent immunohistochemistry staining, which was performed using vimentin (to assess the evolution of the EMT process), Ki-67 (for evaluation of tumor proliferation) and cluster of differentiation 34 (CD34) (for evaluation of angiogenesis) antibodies. The tumor stage, grade, vascular invasion, margin status, rate of expression of the vimentin filaments, microvessel density-CD34 and proliferation rate data were obtained. Finally, the association between the expression of the vimentin filaments and those parameters was resolved statistically. A significant association was shown between the overexpression of the vimentin filaments and tumor size $(\mathrm{r}=0.71, \mathrm{P}=0.03)$, tumor grade $(\mathrm{r}=0.80, \mathrm{P}=0.021)$, angiogenesis $(\mathrm{r}=0.57, \mathrm{P}=0.043)$, proliferation coefficient $(\mathrm{r}=0.06, \mathrm{P}=0.001)$ and vascular invasion $(\mathrm{r}=0.76, \mathrm{P}=0.043)$. Vimentin overexpression did not statistically correlate with the tumor stage or the margin status. Similar to the findings of the present study, certain recent studies have indicated that vimentin filament expression in HBC and CMMGTs is associated with the
\end{abstract}

Correspondence to: Dr Ahad Muhammadnejad, Cancer Research Center, Cancer Institute of Iran, Tehran University of Medical Science, Keshavarz Blvd, Tehran 1419733141, Iran

E-mail:mohamadnajad@yahoo.com

Key words: mammary neoplasms, breast neoplasms, intermediate filaments, epithelial-mesenchymal transition severity of cancer. Thus, spontaneous canine mammary tumor models appear to be an appropriate animal model for breast cancer research, and the results of the present study could aid to reinforce the association.

\section{Introduction}

Canine malignant mammary gland tumors (CMMGTs) are the most common malignancies observed in females, with a low post-surgery overall survival rate $\operatorname{COSR}(1,2)$. Statistical analyses indicate that in canine mammary gland tumors, the recurrence risks of malignant tumors are 13-fold higher than benign tumors (3). Currently, numerous studies are being conducted on CMMGTs and it has been revealed that the biological behavior of CMMGTs is similar to that of the human breast cancer (HBC) (4). The development of molecular profiling in cancer allows the identification of significant molecular pathways and the description of novel biomarkers (5).

Biomarkers have specific roles in the determination of OSR and are recognized as a novel targeted therapy. Therefore, the assessment of biomarkers is considered to be useful in cancer research. By contrast, novel molecular pathways have aided in achieving therapeutic goals. Thus far, numerous significant molecular pathways in mammary gland tumors have been recognized and are being extensively studied (4). As aforementioned, there are similarities between HBC and CMMGTs. Therefore, CMMGTs are considered to be an appropriate model for the development of recombinant drugs and monoclonal antibodies for the treatment of HBC (6). Tumoral xenograft models have been recommended as the preclinical phase of cancer, however, increasing evidence suggests that spontaneous CMMGTs have more similarities with HBC (3).

In recent years, the vimentin biomarker has been extensively investigated in cancers. The overexpression of vimentin filaments in types of cancer of an epithelial origin is abnormal and represents phenotypic changes of malignant epithelial cells into mesenchymal cells, known as epithelial-mesenchymal 
transition (EMT) (7). Although EMT has been identified to occur during embryogenesis and wound healing, its role in cancers of an epithelial origin, along with some of its mechanisms, has been recently identified (8). There is numerous evidence indicating that overexpression of EMT-related genes in the epithelial malignant cells increases the invasiveness and metastatic potential $(7,8)$. Furthermore, the findings of HBC studies have demonstrated that overexpression of the vimentin filaments in breast malignant cells correlates with a poor prognosis and that EMT leads to unfavorable clinicopathological features in patients (5).

Currently, multiple biomarkers are applied for detection, prognosis and prediction of cancer. Based on the numerous similarities between CMMGTs and HBC, a previous study has examined the accuracy of the markers for CMMGTs (9).

The present study aimed to assess the correlation between vimentin overexpression and the clinicopathological features of CMMGTs, and to validate the associated CMMGT model.

\section{Materials and methods}

CMMGTs and immunohistochemistry (IHC) analysis. In the retrospective observational study, the investigators were blinded to all the pathological diagnostic stages. A total of 42 CMMGTs obtained from bitches that underwent surgery in several small animal clinics in Tehran (Iran) between 2009 and 2013 were collected and studied. The inclusion criteria were CMMGTs that adhered to the Goldschmidt et al (10) guideline, with histology test results of malignant epithelial neoplasms or special types of malignant epithelial neoplasms; CMMGTs with complete clinical data; and tumors in the initial stage. Recurrent tumors were excluded from the study. All the recorded clinical data, including the age of the canines, tumor size, affected area of the breast, lymph node involvement or recognizable metastases, and the presence or absence of invasion, were collected. Paraffin sections or blocks of the specimens were prepared and the slides were stained with hematoxylin and eosin (H\&E) and observed under a light microscope to confirm the malignancy of tumors. Blocks, $4-\mu \mathrm{m}$ thick, underwent IHC staining performed with vimentin (clone: Vim 3B4; Dako, Carpinteria, CA, USA), Ki-67 (clone: MIB-1; Dako) and cluster of differentiation 34 (CD34) (clone: QBEnd 10; Dako) antibodies according to the manufacturer's instructions.

Clinical evaluation. The tumor size of the tissues were examined and classified as $\mathrm{T} 1, \mathrm{~T} 2$ and $\mathrm{T} 3(\mathrm{~T} 1<3, \mathrm{~T} 2=3-5$ and $\mathrm{T} 3>5 \mathrm{~cm}$ ) according to the criteria recommended by Cassali et al (9). The regional lymph nodes were classified as N0, N1 and N2 according to the classification developed by Cassali et al (9). The clinical cancer stages (stages I-IV) [tumor-node-metastasis (TNM)] were determined according to the system described by Cassali et al (9).

Histological analysis. The tumors were graded from I to III using a guideline based on the study by Goldschmidt et al (10), as well as tubule formation, nuclear pleomorphism and mitotic index (9). Vascular invasion was considered positive if the H\&E staining revealed clear malignant cells in the vessels. The margin status of the paraffin blocks subjected to $\mathrm{H} \& \mathrm{E}$ staining was investigated and all the specimens were classified as free (0) and involved (+1) based on the absence or presence of the deep margin, respectively.

The rate of membrane immunoreactivity of the vimentin filaments in the malignant epithelial cells was interpreted according to the intensity and degree of reactivity. The canine fibrosarcoma tumor was used as the positive control. The pathologist randomly selected 10 fields and rated the intensity and degree of reactivity under magnification, $\mathrm{x} 400$, based on the positive control. A three-tiered scoring system was conducted as 0 (negative), +1 (weak positive) and +2 (strong positive) (11).

Angiogenesis evaluations were performed as previously described by Dhakal et al (12) and da Silva et al (13) for the microvessel density (MVD)-CD34 by counting the number of endothelial cells and immunoreactive lumens in four hot-spot regions under magnification, $\mathrm{x} 100$, and determining the average value. The results were reported as negative (0; MVD $<20)$, weak positive $(+1 ; 20 \leq \mathrm{MVD} \leq 40)$ and strong positive $(+2 ; \mathrm{MVD}>40)(12,13)$.

For the evaluation of tumor proliferation using the Ki-67 antibody, 10 fields showing 100 malignant epithelial cells were randomly selected and the number of immunoreactive nucleoli was determined under magnification, $\mathrm{x} 400$. The proliferation index results were reported as percentages [negative $(0),<5$; weak positive (+1), 5-15; strong positive (+2), >15\%] (9).

Statistical analysis. The results are presented as mean \pm standard error of the mean. The ordinal scale was employed for the measurement of medians. Linear regression was used to determine the correlation between the overexpression of the vimentin filaments and tumor size, tumor stage, tumor grade, vascular invasion, margin status, tumor angiogenesis and tumor proliferation. All the statistical analyses were performed using BioStat ${ }^{\circledR} 2008$ software (AnalystSoft, Inc., Vancouver, $\mathrm{BC}$, Canada). $\mathrm{P}<0.05$ was considered to indicate a statistically significant difference.

\section{Results}

Tumor stage and IHC analyses. The mean age of the canines included in the present study was $7.6 \pm 0.9$ years and there were $35(83.2 \%)$ tumors involved the abdominal region. In terms of the involvement of the mammary glands, $29(69.0 \%)$ had only one mammary gland involved. In addition, mamectomy (40.5\%) was the most common surgery technique. The tumor sizes were $\mathrm{T} 1, \mathrm{~T} 2$ and $\mathrm{T} 3$ at $47.6,42.8$ and $9.6 \%$, respectively. According to the TNM system, tumor staging showed that stage I tumors had a frequency of $33.3 \%$ and stage I tumors, which were the most common clinical stage II noted in the present study, had a frequency of $52.4 \%$ (Table I). Based on the guideline recommended by Goldschmidt et al (10), the histopathological findings revealed that $59.5 \%$ of the tumors were simple carcinoma. The other histological features are shown in Fig. 1. The grade II tumors were observed most frequently with a frequency of $54.8 \%$. Furthermore, $45.8 \%$ of the tumors had margin involvement and a positive vascular invasion was reported in $52.4 \%$ of the tumors (Table I).

The IHC results showed that $12(28.6 \%)$ and six (14.3\%) of the specimens were strong- and weak-positive for the vimentin biomarker, respectively. The findings of Ki-67 and 
Table I. Clinical and pathological results of canine malignant mammary gland tumors in the present study.

\begin{tabular}{|c|c|c|c|}
\hline \multirow[b]{2}{*}{$\begin{array}{l}\text { Variables }(n=42) \\
\text { score }\end{array}$} & \multicolumn{2}{|c|}{ Vimentin } & \multirow[b]{2}{*}{ P-value } \\
\hline & $\begin{array}{l}\text { Positive }^{\mathrm{a}} \\
(\mathrm{n}=18)\end{array}$ & $\begin{array}{l}\text { Negative } \\
(n=24)\end{array}$ & \\
\hline \multicolumn{4}{|l|}{ Grade } \\
\hline I & 2 & 10 & \multirow[t]{3}{*}{0.021} \\
\hline II & 10 & 13 & \\
\hline III & 6 & 1 & \\
\hline \multicolumn{4}{|l|}{ Stage } \\
\hline I & 7 & 9 & \multirow[t]{4}{*}{ NS } \\
\hline II & 8 & 13 & \\
\hline III & 3 & 2 & \\
\hline IV & 0 & 0 & \\
\hline \multicolumn{4}{|l|}{ Tumor size } \\
\hline $\mathrm{T} 1$ & 7 & 13 & \multirow[t]{3}{*}{0.030} \\
\hline $\mathrm{T} 2$ & 8 & 10 & \\
\hline $\mathrm{T} 3$ & 3 & 1 & \\
\hline \multicolumn{4}{|l|}{ Margin status } \\
\hline Free & 6 & 17 & \multirow[t]{2}{*}{ NS } \\
\hline Involved & 12 & 7 & \\
\hline \multicolumn{4}{|l|}{ Vascular invasion } \\
\hline Present & 14 & 10 & \multirow[t]{2}{*}{0.043} \\
\hline Not present & 4 & 14 & \\
\hline \multicolumn{4}{|l|}{$\mathrm{Ki}-67$} \\
\hline Low & 3 & 13 & \multirow[t]{3}{*}{0.001} \\
\hline Moderate & 6 & 8 & \\
\hline High & 9 & 3 & \\
\hline \multicolumn{4}{|l|}{ MVD-CD34 } \\
\hline Low & 1 & 8 & \multirow[t]{3}{*}{0.043} \\
\hline Moderate & 5 & 15 & \\
\hline High & 12 & 1 & \\
\hline
\end{tabular}

${ }^{a}$ Weak and strong. NS, not significant; MVD, microvessel density; CD34, cluster of differentiation 34 .

MVD-CD34 analyses are illustrated in Table I. Although the statistical analysis represented a significant association between vimentin filaments overexpression and the tumor size $(\mathrm{r}=0.71, \mathrm{P}=0.03)$, tumor grade $(\mathrm{r}=0.80, \mathrm{P}=0.021)$, angiogenesis $(r=0.57, P=0.043)$, proliferation coefficient $(r=0.06$, $\mathrm{P}=0.001)$ and vascular invasion $(\mathrm{r}=0.76, \mathrm{P}=0.043)$, there was no statistical correlation between the vimentin filaments and the cancer stage and margin status (Tables I and II).

\section{Discussion}

The result of the present study revealed that the overexpression of the vimentin filaments was associated with numerous clinicopathological features observed in tumors with unfavorable characteristics. Some studies have demonstrated that the presence of vimentin biomarkers is linked to a poor prognosis in $\operatorname{HBC}(7,11,14)$. The study by Hemalatha et al (11) indicated
Table II. Correlation of vimentin filaments expression with clinocopathological features of the canine malignant mammary. ${ }^{\mathrm{a}}$

Vimentin filaments expression

\begin{tabular}{ll}
\hline Tumor staging & - \\
Tumor grading & + \\
Tumor size & + \\
Tumor margin status & - \\
Vascular invasion & + \\
Tumor proliferation & + \\
Tumor angiogenesis & + \\
\hline
\end{tabular}

aData are presented as positive (+) and negative (-).

\section{Histotypes}

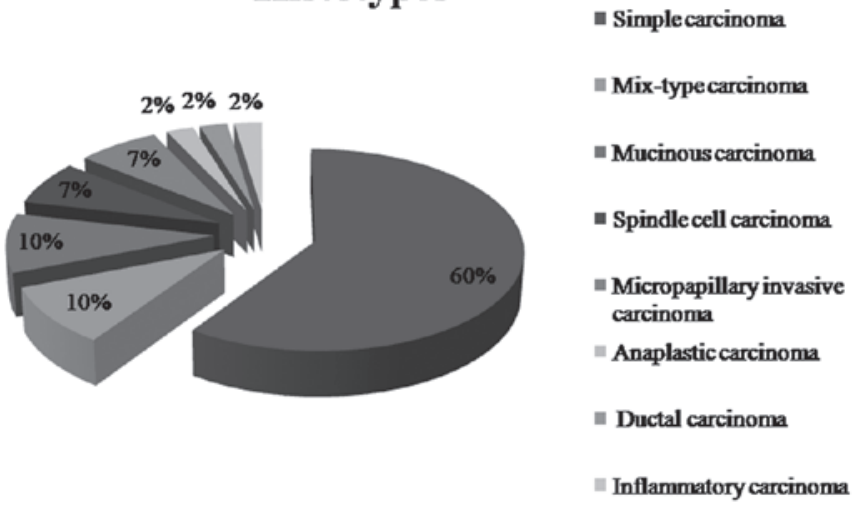

Figure 1. Occurrence rate of various histotypes of canine malignant mammary gland tumors. According to the Goldschmidt histological system, $\sim 60 \%$ of the tumors were simple carcinoma and three others, which were anaplastic, ductal and inflammatory carcinomas, had the lowest occurrence rate.

that overexpression of vimentin is not associated with tumor grade and Ki-67, whereas Vora et al (14) concluded that the invasion potency is increased by the overexpression of vimentin and the loss of cytokeratin in breast cancer. However, Yamashita et al (7) evaluated the expression of vimentin in invasive ductal carcinoma of sporadic breast cancer and revealed that the overexpression of vimentin increased in the subtype of triple-negative breast cancer and was associated with invasiveness potency (8).

EMT is a process in which the epithelial cells lose their epithelial characteristics and acquire mesenchymal characteristics. This mechanism occurs in breast cancer and results in the reduction or loss of cadherin adhesion molecules, loss of luminal cytokeratin, enhancement of basal cytokeratin, manifestation of different proteins, including $\alpha$-smooth muscle actin, changes in the expression of matrix metalloproteinase genes and transforming growth factor- $\beta$ (TGF- $\beta$ ) and increased expression of vimentin, thus facilitating migration and metastasis of malignant cells (14-16).

The evaluation of vimentin expression in breast cancer is considered to be beneficial for determining the prognosis. Furthermore, analysis of the vimentin expression has therapeutic value as the tumor progression may be controlled if vimentin and its upstream line are targeted (17) and such 
mechanisms would be traceable in CMMGTs. In the study by Yoshida et al (16) on a canine mammary cancer cell line, it was concluded that an increase in TGF- $\beta$ induced vimentin expression and increased invasion potency. Krol et al (18) examined five cell lines in canine mammary cancer and found that the cell culture of these cell lines underwent genetic alterations favoring EMT. Terra et al (19) analyzed benign and malignant canine mammary glands and observed that vimentin overexpression had a direct association with the malignancy of tumors.

Numerous studies on vimentin demonstrated that EMT occurs in humans, as well as canines, and that vimentin expression is associated with tumor malignancy $(14,15,17)$. However, unlike the previous studies, in the present study, no association was observed between vimentin expression and cancer stage, similar to the study by Terra et al (19). In addition, there was no correlation between vimentin expression and margin status, thus warranting further studies in this area.

Similar to the findings of the present study, certain recent studies have indicated that vimentin filament expression in HBC and CMMGTs is associated with the severity of cancer. Thus far, numerous studies have demonstrated the similarity between HBC and CMMGTs and a majority of them have concluded that the biological behavior of CMMGTs is similar to that of HBC $(3,4,6)$. Pinho et al $(6)$ indicated that CMMGTs can be regarded as an HBC animal model and that HBC and CMMGTs have similar biomarkers, thus making the canine mammary tumor an appropriate model for epidemiological, genetic and therapeutic studies on HBC (4).

Although there are breast xenograft models, there appears to be a gap between the in vivo and in vitro models, and canine mammary gland tumor models could be the appropriate choice. Several studies have supported the association between the biomarkers and clinicopathological characteristics of HBC and CMMGTs $(3,4)$. The study by Muhammadnejad et al (3) indicated that HER-2/neu changes in CMMGTs are similar to those in HBC and Queiroga et al (4) have also investigated this association.

Thus, spontaneous canine mammary tumor models appear to be an appropriate animal model for breast cancer research and the results of the present study could aid to reinforce the model. To make advancement in the treatment of canine cancers, vimentin could be targeted in the CMMGT models in the future. In addition, the results obtained could be helpful in the treatment of HBC. However, ethical issues should be considered in modeling studies and the 3Rs (reduction, refinement and replacement) should be considered in canine oncology studies. In addition, the oncology studies designed should also be in aid of the treatment for cancers in canines.

\section{Acknowledgements}

The authors express their gratitude to Tehran Veterinary Hospital and the Small Animal Hospital of Faculty of Veterinary Medicine of Tehran University. Also, thanks to Dr E'temad Moghaddam, Pathology Lab, and Miss Morsali for their great technical support for the IHC staining.

\section{References}

1. Santos AA, Lopes CC, Ribeiro JR, Martins LR, Santos JC, Amorim IF, et al: Identification of prognostic factors in canine mammary malignant tumours: a multivariable survival study. BMC Vet Res 9: 1, 2013.

2. Karayannopoulou M, Kaldrymidou E, Constantinidis TC and Dessiris A: Adjuvant post-operative chemotherapy in bitches with mammary cancer. J Vet Med A Physiol Pathol Clin Med 48: 85-96, 2001.

3. Muhammadnejad A, Keyhani E, Mortazavi P, Behjati F and Haghdoost IS: Overexpression of HER-2/neu in malignant mammary tumors; translation of clinicopathological features from dog to human. Asian Pac J Cancer Prev 13: 6415-6421, 2012.

4. Queiroga FL, Raposo T, Caravalho MI, Prada J and Pires I: Canine mammary tumours as a model to study human breast cancer: most recent findings. In Vivo 25: 455-465, 2011.

5. Karihtala P, Auvinen P, Kauppila S, Haapasaari KM, Jukkola-Vuorinen A and Soini Y: Vimentin, zeb1 and Sip1 are up-regulated in triple-negative and basal-like breast cancers: association with an aggressive tumour phenotype. Breast Cancer Res Treat 138: 81-90, 2013.

6. Pinho SS, Carvalho S, Cabral J, Reis CA and Gärtner F: Canine tumors: a spontaneous animal model of human carcinogenesis. Transl Res 159: 165-172, 2012.

7. Yamashita N, Tokunaga E, Kitao H, Hisamatsu Y, Taketani K, Akiyoshi S, et al: Vimentin as a poor prognostic factor for triple-negative breast cancer. J Cancer Res Clin Oncol 139: 739-746, 2013.

8. Kokkinos MI, Wafai R, Wong MK, Newgreen DF, Thompson EW and Waltham M: Vimentin and epithelial-mesenchymal transition in human breast cancer - observations in vitro and in vivo. Cells Tissues Organs 185: 191-203, 2007.

9. Cassali GD, Lavalle GE, De Nardi AB, Ferreira E, Bertagnolli AC, Estrela-Lima A, et al: Consensus for the diagnosis, prognosis and treatment of canine mammary tumors. Braz J Vet Pathol 4: 153-180, 2011.

10. Goldschmidt M, Pena L, Rasotto R and Zappulli V: Classification and grading of canine mammary tumors. Vet Pathol 48: 117-131, 2011.

11. Hemalatha A, Suresh TN and Kumar ML: Expression of vimentin in breast carcinoma, its correlation with Ki67 and other histopathological parameters. Indian J Cancer 50: 189-194, 2013.

12. Dhakal HP, Bassarova A, Naume B, Synnestvedt M, Borgen E, Kaaresen $\mathrm{R}$, et al: Breast carcinoma vascularity: a comparison of manual microvessel count and Chalkley count. Histol Histopathol 24: 1049-1059, 2009.

13. da Silva BB, Lopes-Costa PV, dos Santos AR, de Sousa-Júnior EC, Alencar AP, Pires CG, et al: Comparison of three vascular endothelial markers in the evaluation of microvessel density in breast cancer. Eur J Gynaecol Oncol 30: 285-288, 2009

14. Vora HH, Patel NA, Rajvik KN, Mehta SV, Brahmbhatt BV, Shah MJ, et al: Cytokeratin and vimentin expression in breast cancer. Int J Biol Markers 24: 38-46, 2009.

15. Moustakas A and Heldin P: TGF $\beta$ and matrix-regulated epithelial to mesenchymal transition. Biochim Biophys Acta: Feb 18, 2014 (Epub ahead of print).

16. Yoshida K, Saito T, Kamida A, Matsumoto K, Saeki K, Mochizuki M, et al: Transforming growth factor- $\beta$ transiently induces vimentin expression and invasive capacity in a canine mammary gland tumor cell line. Res Vet Sci 94: 539-541, 2013.

17. Satelli A and Li S: Vimentin in cancer and its potential as a molecular target for cancer therapy. Cell Mol Life Sci 68: 3033-3046, 2011.

18. Król M, Pawłowski KM, Szyszko K, Maciejewski H, Dolka I, Manuali E, Jank M and Motyl T: The gene expression profiles of canine mammary cancer cells grown with carcinoma-associated fibroblasts (CAFs) as a co-culture in vitro. BMC Vet Res 8: 35, 2012.

19. Terra EM, Magalhães GM, Rodrigues MP, Amorim RL, Rocha NS and Costa MT: Immunohistochemical expression of TGF $\beta$, E-cadherin and vimentin in benign and malignant neoplasias of canine mammary gland. BMC Proceedings 7 (Suppl 2): P20, 2013. 